\title{
Douglas G. McNeel: stay current reading research literature, there is no substitute for hard work!
}

Submitted Sep 03, 2018. Accepted for publication Sep 04, 2018.

doi: $10.21037 /$ cco.2018.09.01

View this article at: http://dx.doi.org/10.21037/cco.2018.09.01

\section{Editor's note}

About 1 out of 9 men will be diagnosed with prostate cancer during his lifetime. It occurs most frequently in men aged 65 or older. In America, prostate cancer is the most common cancer other than skin cancer, and is the second leading cause of cancer death in the male population. According to the statistics of The American Cancer Society, over 164,000 new cases of and over 29,000 deaths from prostate cancer are estimated for 2018 (1). While the risk of developing cancer depends on various factors, such as age, genetics, and exposure to risk factors, the incidence of prostate cancer is not obviously linked to any preventable risk factors (2). This has led the scientific world to conduct more in-depth studies into the molecular biology and immunology of the cancer.

Over the past two decades, Dr. Douglas G. McNeel from the Department of Medicine at the University of Wisconsin (Figure 1) has been making sustained effort in studying prostate cancer immunology with an aim to develop effective anti-tumor vaccines to treat prostate cancer. His team has specifically focused on DNA vaccines as T cell activating therapies with research aiming to understand their mechanisms of action and resistance. Chinese Clinical Oncology (CCO) is honored to interview Dr. McNeel, who will share with us what he regards as the critical issues facing the field of prostate cancer immunology, the significance of his proposed rodent model in the evaluation of immunological treatments, the current funded research project that he is involved in, and his candid advice to young researchers in developing their career.

\section{Expert's introduction}

Douglas McNeel, MD, PhD, currently serves as the Professor of Medicine at the University of Wisconsin, Madison, the US. He is a genitourinary medical oncologist with a laboratory and clinical research program focusing

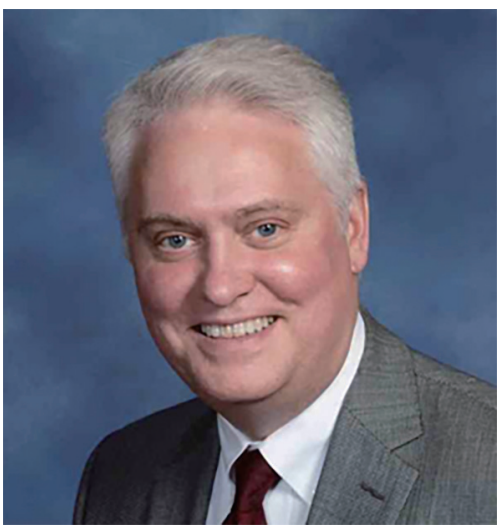

Figure 1 Dr. Douglas G. McNeel.

on prostate cancer immunology. The long-term goal of these efforts is to develop effective anti-tumor vaccines as treatments for prostate cancer. His current research efforts seek to identify immunologically recognized proteins of the prostate, characterize these as tumor target antigens, evaluate anti-tumor genetic vaccines targeting these antigens in appropriate pre-clinical models, specifically evaluating plasmid DNA vaccines encoding these antigens, and translate these findings to early phase human clinical trials.

Dr. McNeel's work has been supported by multiple grants from the National Institutes of Health (NIH), the Department of Defense Prostate Cancer Research Program, and the Prostate Cancer Foundation. Besides, he holds many patents and investigational new drug applications and has written and conducted multiple investigator-initiated clinical trials to pursue translational directions identified by his laboratory. Dr. McNeel is a member of multiple professional societies, including American Association for Cancer Research, American Association of Immunologists, American Society of Clinical Oncologists, and the Society for Immunotherapy of Cancer. He is also an Editorial Board Member of $C C O$. 


\section{Interview}

CCO: Having been studying in the field of prostate cancer immunology for almost 2 decades, what do you think are the critical issues facing the field right now?

Dr. McNeel: Critical issues arise from the observation that the majority of patients with prostate cancer do not respond to T-cell checkpoint blockade therapies, notably targeting cytotoxic T-lymphocyte-associated antigen 4 (CTLA-4) or programmed cell death 1 (PD-1). This has been attributed to the relative paucity of tumor-infiltrating lymphocytes in prostate tumors and a low mutation burden in this disease. However, it is apparent that $\mathrm{T}$ cell responses to prostate cancer associated antigens are relatively common in the peripheral blood of patients with prostate cancer. In addition, vaccines have demonstrated activity, and one vaccine is an approved therapy for prostate cancer, suggesting that approaches to augment T-cell immunity to prostate cancer can be beneficial. So, a critical issue is understanding the prostate cancer tissue microenvironment to understand if there are differences compared with other tumors permitting/excluding T-cell infiltration and affecting intratumoral $\mathrm{T}$-cell function.

CCO: In your previous study, you proposed a prostate cancer tumor model in Lewis rats for evaluation of immunological treatments. What is the significance of this model?

Dr. McNeel: We had developed this model to specifically evaluate prostatic acid phosphatase (PAP) as a model antigen, given that rats, unlike mice, express a prostatespecific acid phosphatase nearly identical to human PAP. In addition, rat prostate tumors driven by simian virus 40 (SV40) under a prostate-specific promoter, similar to the murine TRAMP tumor model, remain adenocarcinomas even following androgen deprivation, unlike the murine model. Hence, the rat model served as a better model of human prostate cancer in some ways. However, given other immunological agents that are specific for mice, we have moved many of our preclinical studies to murine models rather than use this rat model.

CCO: Researchers often encounter a problem that it's hard to find a cutoff point where animal models can be extrapolated to human trials. What is the situation in your own research?

Dr. McNeel: The aim of our research has been to develop vaccines as therapies for human prostate cancer, and in that context learn about human tumor immunology. We have used animal models to understand basic principles, evaluate safety and anti-tumor efficacy, and develop the tools for measuring immune effect following vaccination. Ultimately, no model is a substitute for a human trial evaluation, particularly as our goal is to develop therapies that can make a difference for patients with cancer, and we can't learn about human immunology without clinical trials. Hence, with evidence of safety and anti-tumor effect in appropriate animal models, being able to measure the biological effect, and understanding the mechanism of action, we have attempted to move these approaches directly to clinical trials. We have been successful in this approach and have now conducted several clinical trials using approaches and vaccines developed in the laboratory and evaluated in rodent models.

\section{CCO: In your opinion, what is the most promising anti- tumor genetic vaccine in the rodent models?}

Dr. McNeel: Our focus has been on DNA vaccines, given their simplicity and safety. We have been using rodent models to evaluate their efficacy, study their mechanism of action, and understand mechanisms of tumor resistance. We have translated this approach to clinical trials using DNA vaccines encoding either PAP or a portion of the androgen receptor (AR). Both vaccines have demonstrated immunological effect and some anti-tumor effects. Hence, we believe that both of these vaccines (pTVG-HP and pTVG-AR) are promising candidates for further evaluation, particularly in combination with agents targeting mechanisms of tumor resistance.

CCO: Your studies have been holding research grants from NIH and some other institutions. Would you introduce us to a recent funded research project that you are involved in?

Dr. McNeel: We have had research funding from several sources, including the US NIH, the US Department of Defense Prostate Cancer Research Program, and the Prostate Cancer Foundation. These cover a wide range of topics, and for most I have served as principle investigator. All have focused on prostate cancer immunology. In one example, we had observed that CD8+ T cells activated with vaccination express $\mathrm{PD}-1$. The strength of the 
activation signal affected the duration of PD-1 expression, and if prolonged, led to an inferior anti-tumor response. Combining vaccination with PD-1 blockade gave a greater anti-tumor response. With funding from one source we evaluated this approach in a human clinical trial, evaluating the timing of PD-1 blockade with vaccination in patients with advanced prostate cancer. With funding from another source, we have been dissecting the mechanism in preclinical models, and determining whether other agents can affect PD-1 expression following T-cell activation, and whether other T-cell regulatory molecules are similarly affected.

\section{CCO: What do you regard as the most difficult aspects of research?}

Dr. McNeel: Laboratory and clinical trial research are costly, and the rising costs are outpacing the resources available from grants. Hence, the most difficult aspect of research is continuing to find sufficient funding.

\section{CCO: Out of a variety of medical disciplines, why are you particularly interested in the field of cancer immunology?}

Dr. McNeel: I was drawn to cancer research very early in my career because it was such an obvious huge needtoo many people die from cancer each year. And there were so many directions that needed to be pursued, and I wanted a career in which I had one foot in the clinic and one foot in the laboratory. It was also clear to me that most cancers are adaptive diseases, always in evolution. Thus, I was more interested during my training in studying cancer immunology and cancer vaccines, as these are treatments that could potentially elicit an adaptive response. While more challenging, this was of more interest to me than studying, for example, individual agents and pathways that tumors could circumvent. It has been rewarding to see the renewed interest and successes of cancer immunotherapy treatments over the last several years.

Cite this article as: Li B. Douglas G. McNeel: stay current reading research literature, there is no substitute for hard work! Chin Clin Oncol 2018;7(6):63. doi: 10.21037/cco.2018.09.01
CCO: Having mentored many trainees over the years, what do you usually remind them to do if they want to become successful in research?

Dr. McNeel: First, there is no substitute for hard work. Many people have good ideas, but the reality of research is that many lines of research will not lead anywhere. Hence, it is often necessary to pursue several lines of research at the same time, and that takes time and effort. Second, I encourage people to set goals and deadlines. After pursuing some areas for a long time, it is often difficult to know when to stop if a direction is not successful, and it's easier to set deadlines in advance. Third, you have to stay current reading the research literature. Finally, I learned from my own doctoral advisor that it is more rewarding to follow the science and address the questions that arise from the research, rather than develop a method and use that to answer specific questions.

\section{Acknowledgements}

We would like to express our sincerest gratitude to Dr. Douglas G. McNeel for sharing his insights and opinions with us.

\section{Footnote}

Conflicts of Interest: The author has no conflicts of interest to declare.

\section{References}

1. American Cancer Society. Key Statistics for Prostate Cancer. 2018. Available online: https://www.cancer.org/ cancer/prostate-cancer/about/key-statistics.html

2. Cancer Research UK. Prostate cancer statistics. 2018. Available online: https://www.cancerresearchuk.org/ health-professional/cancer-statistics/statistics-by-cancertype/prostate-cancer

(Science Editor: Brad Li, CCO, editor@thecco.net) 